\title{
Mental health problems and suicide in the younger generation - implications for prevention in the Navy and merchant fleet
}

Vsevolod Rozanov

Saint-Petersburg State University, Saint-Petersburg, Russian Federation

\begin{abstract}
Psychologists and psychiatrists worldwide are expressing concerns regarding the growing prevalence of mental health problems and the incidence of suicide in young adults. The reasons are seen in the extremely high tempo of social changes, information pressure, and values evolution in the younger generations, which are exposed to growing inequalities, loneliness and lack of social support. Poverty, social isolation, consumerism, hedonism, and unrealistic expectations of the future generate in the vulnerable part of the young adults inevitable frustrations, which give way to depression, anxiety, addictions, and suicide. This creates additional risks for the situation on board ships, both military and merchant, and requires greater efforts during pre-admission selection and in the course of the service or voyages. Suicides in the Navy are better registered than in the cargo fleet and are lower than in the same age and gender groups from the general population, and usually lower than in other types of forces. Data on suicides in the civilian maritime sector are less conclusive, but suggest it as a growing problem, especially considering stress on board. Recent studies revealed quite a lot of mental health problems in the merchant fleet crews, including depression, anxiety, and suicidal thoughts. Among the reasons such factor as "flag of convenience" strategy that implies lower standards, recruiting of the less trained and lower-paid workforce, multinational and multilanguage rotating crews, higher workload and stress and insufficient level of the pre-employment medical examination are mentioned. Recent trends in the mental health of the youth demand higher awareness both in the military ships and in the merchant fleet. We consider that more education and training aimed at mental health problems identification and stress-resilience promotion are needed both for the military and civilian staff on board. Better education of the whole personnel and "healthy ship" approach (better recognition of the crew members' needs, attention to mental health problems, nutrition, physical activity, etc.) may be applied both for the Navy and merchant fleet.
\end{abstract}

(Int Marit Health 2020; 71, 1: 34-41)

Key words: mental health problems, suicides, young adults, suicide prevention, Navy, merchant fleet

\section{INTRODUCTION}

The world has changed a lot for the last decades, and there is a general feeling that today unprecedented global forces are shaping the health and wellbeing of the largest generation of 10 to 24-year-olds (the so-called iGen, or Generation Z) in human history. Specialists in mental health promotion and psychiatrists worldwide are expressing concerns regarding the growth of the problems of mental health in young adults. According to the most recent epidemiological studies, the prevalence of any disorder in younger people is about $20 \%$ for the last year [1-3]. Among the disorders, there are anxiety, depression, addictions, hyperactivity, psychosomatic symptoms, personality disorders, behavioural problems, and phobias. Studies and reviews provide evidence that in recent decades the prevalence of anxiety, depression and substance abuse 
in adolescents and young adults has increased most of all [4-6]. Moreover, birth cohorts comparisons testify that some personality traits, for instance, neuroticism and clinical scales of MMPI, have grown substantially too [7]. Mental health problems are more pronounced in youngsters from lower social-economic strata, though they are inherent to all social classes. Comorbidity of disorders, as well as general and mental health poor rating, is associated with suicidal thoughts and more serious manifestations of suicidal behaviour - attempted and completed suicide [3].

Analysis of the situation with suicides in adolescents and young adults shows that suicides in this contingent are growing worldwide $[8,9]$. The peak in the most economically developed countries has passed, but even in the countries, where national suicide prevention programmes were implemented, suicide levels did not drop substantially. In the 90 s a rise in suicide rates in young adults has been very typical to Britain, Australia, and Western America, then the focus shifted to post-soviet countries, and recently have touched Latin America, China, Korea and other countries of South-East Asia [9, 10]. It may be hypothesized that recent decades rise in rates in the countries and communities with traditional cultures are the results of the pressure of the postmodernism that promotes very liberal attitudes to life and death.

\section{MILITARY ENVIRONMENT AS A MODEL FOR A MERCHANT FLEET}

Young soldiers constitute the majority of personnel in conscription armies, many contracting specialists in the Navy are also young adults who have just graduated from military colleges or high schools. No surprise that when the time comes for the service they may 'import' their risks to the military environment. This raises several important questions: 1) how military forces can protect themselves from drafting personalities with hidden suicidal tendencies; 2) how to prevent exacerbation of mental health problems in the stressful military environment, and 3) what can be done to promote mental health and stress-resilience of the staff. One of the relevant questions is also if the knowledge accumulated within the military and the Navy in particular may be extended to civilian maritime sector given the unifying character of marine occupation with its' organisational and psychological peculiarities.

Suicides in the military may have the same risk factors as in the civilian population, i.e. mental disorder, life stress and trauma, alcohol and drugs abuse, frustrations, financial and legal problems, etc. Speaking about specific factors in the military settings, it was long supposed that combat exposure and traumatic experiences may be main additional reasons for mental health problems and suicides. Nevertheless, one of the recent studies from the United States
(US) has revealed that while suicide rates for soldiers who served in Iraq and Afghanistan more than doubled from 2004 to 2009 to more than 30 suicides per 100,000, the trend among those who were never deployed nearly tripled to between 25 and 30 suicides per 100,000. Rates for similar age and sex civilians remained almost steady at 19 suicides per 100,000 during this time. These results, as the authors say, "argues indirectly against the view that exposure to combat-related trauma is the exclusive cause of the increase in Army suicides", pointing to pre-existing problems among modern youth [11].

Another part of this massive study pointed out that one in four soldiers in the army appears to suffer from at least one psychiatric disorder and one in ten has multiple disorders. Most importantly - about a third of soldiers who attempted suicide had mental disorders that developed before they joined the army: an indication of the general shift towards mental ill-health in young people and a warning that more professional efforts are needed while screening for mental health problems among recruits [12]. The authors discuss that the rise in suicides and diagnoses was the result of two recent US Army trends aimed to recruit or retain personnel. One trend was the liberalisation of screening rules and enrolment of recruits with poor education or conduct records, the other was the practice of forcing soldiers to remain in service beyond their enlistment $[11,12]$. The study did not directly confirm the role of these practices, but in a more recent article dedicated to recruitment problems Mark Perry directly points that efforts to increase military size in US are hampered by poor records of potential recruits, who are undereducated, often obese and with criminal records - the trend that implies also many mental health problems [13].

The situation in different armies and different types of forces may vary. The above-mentioned study may depict specific features inherent to the US Army. On the other hand, military psychologists and psychiatrists from different parts of the world are expressing the same concerns and advocate more efforts during selection and more testing before conscription or signing the contract with a serviceman, both in the Army and the Navy. This is a questionable issue, in some armies, a lot of attention is paid to psychological testing before enrolment, while in others screening for mental health is considered useless and the focus is shifted on performance and hardiness of the serviceman [14-16].

\section{SUICIDES IN THE NAVY - ARE THERE PECULIARITIES?}

Recently, we have tried to outline some common features of suicide within the military environment [17]. The following factors may be associated with an increased risk of suicide in the military context: 1 ) the loss of or lack of personal freedom experienced by people entering rather 
closed and authoritarian system; 2) the masculine culture in many military communities, which may leave little room for self-disclosure and peer support; 3) the risk for personal traumatic stress exposure and subsequent traumatic stress reactions; 4) the easy access to firearms; 5) the military lifestyle with frequent relocations and the break-up of supportive social structures; 6 ) during profound changes in social structures, when downsizing or reorganizing processes in the armed forces take place; and 7) the danger of suicide contagion and clustering of suicides in military units [17].

These specific military environment risk factors may act together with risk factors inherent to the general population (belonging to a vulnerable demographic group, chronic life stress, lack of social support, dysfunctional relations, prior suicide attempt, mental health problems, etc.). Nevertheless, suicide rates in military populations remain, in most cases, lower than in the civilian population of men of the same age. This could be attributed to the existence of protective factors that may counterbalance the situation: 1) the military is a highly organized structure and if the problem is well understood by commanders, prevention programmes may be implemented in a prompt and effective way; 2) there is a preliminary and ongoing medical control of those who are dealing with weapons and certain psychiatric conditions may be recognised early on; 3) special prevention measures may be organized and special means of reporting may be implemented that provide quick identification of suicidal persons along with their referral to specialists; 4) the military can discharge those with suicidal ideations or actions to reduce suicide risk; 5) every case of completed or attempted suicide may be thoroughly investigated producing important information for further prevention models [17].

Navy personnel in all countries that have fleets belongs to the most educated and trained due to high demands existing in the modern battleships and submarines. No surprise that suicides in the Navy are usually rather low. For instance, regular suicide statistics from the US military forces testify that suicide rates in the Navy are the lowest, followed by the Air Force, Army and Marine Corps [18]. Micklewright reported on deliberate self-harm in personnel of the Royal Navy [19] and concluded that these acts should be viewed in the context of the environment that often imposes psychological, emotional and social pressures on servicemen. In the United Kingdom (UK), detailed information on suicide in the regular Armed Forces is published by the Defence Analytical Services Agency (DASA). For the 23 years from 1984 to 2006 , suicide rates in the Army ranged from 12 to 20 per 100,000 , suicide rates in the Naval service ranged from 6 to 14, and from 3 to 15 per 100,000 in the Royal Air Force (RAF), most recent figures (2018) are 10 for the Army, 8 for the Navy and 4 per 100,000 for RAF [20]. Suicide rates in the young age military in the 90 s exceeded rates of the same age and sex group in the general UK population; however, in the recent decades rates in the military are below the corresponding civilian age and sex groups.

Suicide rates vary significantly from country to country, while suicide rates in the military (though usually substantially lower) may be ranked in the same order, which may depict cultural, religious and other nationally resistant peculiarities [17]. On the other hand, time change in the civilian and military environments may be different. As an example, in the former USSR Navy, the time-course changes of suicide rates differed distinctly from the general population: while in the whole USSR there was lowering of suicides in 1986 (preventive effect of the 'perestroika' - democratisation and liberalisation in the country), in the Navy there was a rise in the proportion of suicides in general death structure during the period 1986 to 1995 (period of serious economic problems, fleet downsizing, lowering of the prestige of the marine professions). Only from 1998 to 2000 the percentage of suicides started to diminish. In the Navy, two-thirds of all suicides occurred among conscripted personnel during their first year of service. In $65 \%$ of the cases, the method of suicide was hanging, 20\% firearms, 5.5\% intoxications, 5.2\% self-cutting, $2.2 \%$ jumping and finally, $1.8 \%$ drowning [21].

Though suicide rates in the Navy are low, the impact of such a tragic event on the battleship may be rather high and may induce severe psychological trauma in the crew, especially in the immediate commander-in-charge, or in the closest friends. The whole crew may be also psychologically impacted, depending on the situation. Given this, every case must be thoroughly investigated and a report should be developed according to the existing rules in every Navy, but also a debriefing should be provided for the crew. It applies also to cases when suicide of the crew member happened ashore but became known to the crew.

\section{COMPARISONS WITH THE CARGO FLEET}

The situation in the Navy and cargo fleet definitely has many similarities, especially taking into account conditions on the ships, noise, electromagnetic fields, ship movements, stressful shift work, isolation, lack of social support, remoteness from the family, etc. Moreover, if comparing with other occupations, organisational and psychological factors on merchant ships are more close to the military environment, with high level of subordination, responsibility and crew cohesion. Both in the Navy and merchant ships high demands are imposed on the staff in relation to withstanding austere and dangerous conditions of seafaring, probable fight for the buoyancy and survivability of the ship and during rescue operations.

The merchant fleet, like the Navy service, recruits young educated specialists from corresponding educational institutions, where students are the same representatives of 
generation Z (Digital Natives) with their typical academic stress, loneliness in the social media and lack of social support. They definitely benefit from their advanced digital competency, like managing many tasks at once and high productivity based on abilities of surfing through web, but also may import onboard mental health problems inherent to this generation, including anxiety, depressive thoughts, hyperactivity and impulsivity. Less-educated crew members are even at a bigger risk, especially under current recruiting circumstances. This issue is worth special attention.

Economic factors that are prevailing recently in the cargo fleet seem to produce more mental health risks for workers and employees on board. Globalisation and flags of convenience companies' practices of last decades (lower standards, economic pressure, minimal crews' size, recruitment strategies oriented on lower-paid staff, etc.) changed the global seafarers' trade market, introduced shortened multilingual multinational rotating crews, eliminated medical doctors from the crews, gradually induced higher work-load and caused gaps in seafarers' rights protection [22]. All this satisfies interests of shipping companies' owners, but also provides a fertile ground for conflicts, psycho-social stress, mental health problems, alienation, and impaired social support on board. In confirmation, recent study on seafarers mental health, based on structured interviewing of 1572 merchant ships crew members of different nationalities have revealed that $25 \%$ of respondents may be qualified as having depression higher than in other working and general populations, $17 \%$ may be defined as having heightened anxiety, while $20 \%$ admitted rare and $2 \%$ - constant suicidal thoughts [23]. The working environment has been identified as the main determinant of seafarers' mental health problems, especially such factors as non-caring company culture, violence at work and low job satisfaction. Seafarers from the Philippines and Eastern Europe appeared to be the main victims of workplace violence [23].

Previously in a review of the problems of mental health and suicide among merchant fleet crews authors have outlined two conflicting tendencies that are reflected in the literature reviewed before 2017 [24]. One part of studies, especially older ones, covering period of the second half of the previous century, give an impression of a rather safe picture - pretty low rates of confirmed suicides (1.3-2.2 per 100,000$)[25,26]$, moderate effects of the burn-out syndrome [27], not much complaints on fatigue and distress in the self-reports, higher satisfaction with work and low repatriation rate for mental health reasons [26]. In the Polish seamen and fishermen for 40 years from 1960 to 1999 there were identified 51 suicides, which is quite low in terms of rates given that the population surveyed was 25,000 per year, even if part of cases remained hidden or attributed to other reasons [28]. Though many concerns were expressed regarding stress on the working place on board merchant ships, including unsatisfactory working conditions and psychological strain, the situation with mental health and suicide was not perceived as being worse than in other sectors of economics.

Another part of studies, especially the most recent ones, on the contrary, has reported about growing problems. For instance, studies have found that from $6 \%$ to $35 \%$ of seafarers knew colleagues who considered suicide and pointed that stress, anxiety, depression, and sleep disturbances are serious risk factors among seafarers [24]. Several most recent surveys have confirmed that stress, job strain, and high work demands are directly related to mental health problems among engine officers [29,30] and that seafarers' lifestyles on board (smoking, sedentary behaviour, unhealthy food and lack of physical exercise) may be main contributors to lower well-being [31, 32]. It coincides with the conclusions of the most comprehensive report published up-to-date [24].

What may be the reasons for this negative development in the most recent years? Several explanations may be suggested, among them better understanding of the problem by those in charge for seafarers health and well-being and a shift towards direct questioning about mental health issues and suicidal thoughts (possibly avoided previously due to stigmatisation), which may produce more symptoms. On the other hand one cannot exclude real worsening of the situation, especially when the socio-economic context is analysed. Some authors point on already mentioned fast technological and organisational changes and the increased pressure for economic profitability that may contribute to lower well-being, for example through role conflicts or perceived stress on board [30]. On the other hand, one may agree with the opinion that the complexities of mental health problems identification in multinational and multilingual crews may be the reason [24]. The topic of mental health and suicide remains a strong taboo in many cultures, while modern trends (multinational crews, high workload, the short ship turns, work stress, job insecurity, etc.) elevate the risk of suicides. The problem becomes exposed only during direct questioning within the context of special studies and surveys. Therefore, the issue of mental health and suicides in the merchant fleet crews warrants further research and analysis.

Another systemic problem is seafarers' medical examinations and health assessments - the system is still focused on cardiovascular health, while mental health issues, especially depression and suicide risk remain obscure, either due to low attention from the medical authorities and complexities of objective evaluation, or due to workers' dissimulation of symptoms, which is easy to perform. Moreover, recruiting crews from low-income countries, which is the dominating trend in modern sea trade, often leads to falsification of medical certificates [24]. Therefore, it 
may be noticed, that situation in the cargo fleet has some similarities with the tendencies noticed by US military psychiatrists mentioned above $[11,12]$ and that not enough attention to mental health problems as well as objective difficulties in assessment of suicide risk may contribute to problems in the merchant fleet, while situation in the Navy looks more definite.

It is also important to look at suicide on board in the context of risky behaviours, in terms of 'human factor' and accident analysis [33]. Conditions in which personnel live and work may influence physical and mental health and behaviours, both healthy and unhealthy. People's behaviours and unconscious gestures or motives can be important factors leading to a variety of accidents, from minor traumas to major accidents and deaths. Some genetic factors may be predisposing to risky behaviours while genes-environments covariations may lead to self-selection into risky environments. This may be an additional mechanism through which some individuals may carry their risks with them on board. Another question is how life stress, both in the early periods of life, and further in the working career can exacerbate these risks and lead to suicidal behaviour, and how these types of stress interact [10].

Many interdisciplinary studies are needed to understand better stress-vulnerability and resilience in young people seeking occupation associated with the sea and to develop more predictive tests to prevent those at risk from entering the occupation. It may have a value in the prevention of suicides among seafarers, both in the Navy and in cargo fleet. As to the Navy, though the military system is much more organised and disciplined, some individuals may be seeking additional sensations and stressful experiences, which can contribute to mental health problems and suicide risk. Recent advances in genomic studies and rapid accumulation of reference data may provide more objective tests for suicidality in the future, which may be used in conjunction with psychological testing and psychophysiological evaluations of stress-vulnerability and resilience.

\section{EXPLANATIONS OF YOUTH MENTAL HEALTH PROBLEMS}

One of the most important questions is why above mentioned negative tendencies in mental health and suicides in young adults became so visible in recent decades. Explanatory factors for this phenomenon include quite fundamental features of modern societies, which go beyond inequality and disadvantage. Such factors may be mentioned as modern lifestyles, social and moral conditions associated with high competitiveness, a high value of personal success and unrealistic expectations promoted in young people by the social environment and mass media. The increasing influence of mass media and modern information flows promotes violence, envy, consumerism, fear and social isolation. Other factors that may be named are the weakening of the role of the traditional family, lack of social support, poor parenting, and vague life perspectives [3]. All this means a higher level of psychosocial stress, which is accompanied by anxiety, sleep disturbances, symptoms of depression, reduced performance, or tiredness. Studies evaluate that more than $40 \%$ of youngsters have a feeling of being stressed, and a huge majority of them report they are feeling tiredness in the morning. There are also facts that academic environment and social media in many cases turns into a source of additional distress and anxiety [6].

Such a situation is a reason for serious concerns, especially taking into account that early disturbances may have an impact on the whole life. Adolescence and early adulthood are transitional periods when individuals are particularly sensitive to environmental inputs. Behaviours initiated during adolescence, such as substance use, highrisk sexual behaviour, and risky driving, contribute to poor health outcomes and mortality during later life. Young adults fare worse than adolescents in many areas, with rates of motor vehicle deaths, homicide, substance use, sexually transmitted infections, and mental health problems peaking during young adulthood. Adolescents and young adults altogether seem to have a higher level of certain disorders than older adults; moreover, about $40-50 \%$ of those who have had mental health problems in adolescence may have the same or different problems as being adults [5, 34].

Another important factor is related to meanings and values, and how they are perceived by young people in the society of post-modern. Recent sociological and psychological surveys give evidence, that for several last decades American college students much more often mentioned as "important" such life goal of "being very well off financially" instead of "developing a meaningful philosophy of life". Meaning in life declined in importance by almost half during the 1970s and 1980s, while money and success almost doubled to become the highest-rated goal, and trends have remained relatively stable since $[35,36]$. In most recent decades the same shift is seen in the post-soviet space, India, and China [37]. The growing role of individualism and personal success in opposition to collectivism and social cohesion, growth of hedonism, pragmatism and moral relativism may be the result of the aggressive propagation of these values by mass media [3].

\section{IMPLICATIONS FOR THE NAVY AND MERCHANT FLEET - BUILDING RESILIENCE AND RAISING AWARENESS}

Suicide prevention is a mental health promotion. This formula becomes more and more popular among suicidologists, psychiatrists and mental health providers. The 
essence of the strategy that lays behind it is that we are focusing not only on prevention itself, which implies the identification of risk groups and organising interventions, but addressing wider issues of promotion of resilience and positive functioning in wider populations. This paradigm is based on the concept of mental health, which is a complex non-psychiatric culturally sensitive phenomenon. Modern understanding of mental health includes such factors as emotions, cognition, social functioning, and sense of meaning, purpose in life. It is based on subjective well-being, self-efficacy, autonomy, coping skills, the desire for self-development and social cohesion. So being mentally healthy is something that is far beyond having no mental disturbance [38].

The complexity of the tasks in the modern Navy and merchant fleet means that the cognitive functioning and psychological stability of the staff, from the lowest to the highest level, is crucial. It imposes increased demands on the conscripts, cadets and other specialists, especially in the submarines, deep see ships and highly technological specialised ships. Young people are still joining Navy or merchant fleet to "see the world", but in many cases, this does not happen, instead they are working under stress in front of the computers, being exposed to the same factors as all modern youth, plus inevitable stressful factors of the ship environment. All this means new challenges for the Navy in terms of maintaining high morale, motivation, concentration and cognitive performance of the personnel. Given specific conditions in the Navy (remoteness, stressful environments, high workload, shift work, monotony, high responsibility, etc.) there are higher demands to the stress-resilience and emotional status of any serviceman. During the missions, more specific combat-related stressful factors may appear that may lead to acute operational stress, which in a part of the personnel can over time turn into long-lasting post-traumatic stress disorder. This can be also applied to the merchant ship's crews so far as some of them are exposed to such risks as modern piracy making the atmosphere very close to the military situation, when concerns and fears about possible attack constitute additional stress.

Many authors in military psychiatry and psychology as well as in occupational psychology have formulated important principles of medico-psychological aid, prophylaxis and rehabilitation measures aimed to prevent deferred mental health disturbances and suicides. In some countries, special programs for the Navy are developed, or special psychopathology services are created [39, 40]. In a recent article from Poland, authors are stating that advanced technology on modern ships puts high demands on operators in terms of psychophysical characteristics, mental stability, and cognitive performance. Sailing in dif- ficult conditions, ship movements, austere meteorological conditions, complicated technical equipment, working in isolated groups, functioning within a limited space, noise, vibration, and electromagnetic waves are the factors producing chronic stress. The authors conclude that service on Navy ships requires certain psychophysical qualities and, most important, resilience to stress. They advocate proper selection among the candidates based on psychiatric and psychological counselling for military and medical jurisprudence, as well as better training for doctors and specialists in psycho-prophylaxis of military units in the field of mental hygiene [40]. In the civilian sector it may be transformed into appeal for better training for general practitioners and members of special medical-psychological expert groups involved in crew members medical examinations.

Resilience to stress is an extremely important feature for the Navy personnel, as well as merchant crews' members. According to existing views, it may be both a predisposition and a trained quality. Studies trying to measure biological, psychological, cognitive, behavioural, emotional and spiritual dimensions of resilience have revealed that regulation of fear and anxiety, impulse control and prosocial behaviour may be very important factors. Evaluation of successful servicemen has identified optimism, altruism, moral principles, humour, spirituality, goals in life and constant training as main factors of resilience [41]. There is also a great body of research proving that resilience to stress is the result of the personality development, which implies interventions from rather early childhood, for instance, exposure to mild stress sometimes referred as stress-inoculation [42]. Because of this modern approaches of selection of the staff should pay more attention to all periods of life of the candidate, including early ones. Modern studies of genetics and epigenetics of stress and resilience may soon shed light on intrinsic mechanisms of this phenomenon and possibly may produce a set of more objective predictors that may be assessed before the enrolment of the serviceman or a civilian seafarer [10].

In addition to the better and more focused selection of the staff, one of the issues is improving help-seeking behaviours by young adults in cases of psychological crisis. Military (and Navy in particular) culture may become a barrier for help-seeking when first signs of mental health disturbance or suicidal crisis emerge, thus a policy should be developed that may help to overcome this obstacle. This is absolutely true for merchant ships crews as well, so far as masculine culture is inherent to the maritime profession in general. Multicultural and multilingual cargo ship crews in this respect may exert additional problems due to restricted communication and inability of crew members and officers to notice depression or exacerbation of any other disorder, except of course most severe ones. Studies also suggest 
that improving mental health literacy, reducing stigma, and enhancing the desire of young people for self-reliance may have a positive effect. Much depends also on life goals, meaning in life and self-determination, which is a deep feeling, also depending on the prestige of the seafarers' profession.

Another important practical goal is better training of the personnel, from officers to sailors. In the Navy it may be an additional task for the medical doctor on board [43]. Given limited resources in the civilian sector, better education for all crew members regarding mental health problems, anxiety, signs of depression, stress, crisis, suicidal thoughts, healthy lifestyles, and coping practices should be promoted. The atmosphere of general awareness regarding mental health issues is an important part of the healthy crew. Recent initiatives in this field like creating a Healthy Ship - a healthy lifestyle intervention, encompassing executive health, catering services, medical services, and physical training - give an excellent example that Navy can present to the merchant fleet. This intervention also implies the formation of an effective Unit Health Committee, aimed to promote a healthy lifestyle culture [44]. Many authors call for similar initiatives in the merchant fleet, including health-promoting interventions, physical exercise courses for the crew, smoking cessation, healthy cooking courses, developing positive practices that may strengthen the team spirit, social cohesion, etc. [31, 32]. The challenge, of course, would be to implement these interventions in the conditions on board, especially in the modern cargo ships with rotating crews. On the other hand, much depends on the leadership, and many positive actions may be introduced if there are a will and understanding of the importance of the problem.

\section{CONCLUSIONS}

More attention should be paid to mental health promotion and suicide prevention on board, both in the Navy and the merchant fleet. Not only direct psychological aid, but wider issues like collaboration and mutual goals understanding by officers, medical services, examination and certification system, marine educational system (both naval and civilian), families, servicemen, sea industry workers and employees and trade unions are needed. Three main strategies may be suggested: wider measures that improve general well-being and psychological context together with resilience training, better identification of those at risk together with the development of the atmosphere of confidence and help-seeking, and better education of responsible officers and crew members regarding mental health problems that may enhance early identification and timely support. Many suicides can be prevented and many problems of mental health may be solved if the general level of knowledge and awareness in the Navy and the merchant fleet will be enhanced and if officers and staff will better understand mental health determinants. It should be promoted within the whole marine educational system, both military and civilian, starting from cadets' education.

\section{REFERENCES}

1. Belfer ML. Child and adolescent mental disorders: the magnitude of the problem across the globe. J Child Psychol Psychiatry. 2008; 49(3): 226-236, doi: 10.1111/j.1469-7610.2007.01855.x, indexed in Pubmed: 18221350.

2. Ravens-Sieberer U, Erhart M, Gosch A, et al. European KIDSCREEN Group. Mental health of children and adolescents in 12 European countries-results from the European KIDSCREEN study. Clin Psychol Psychother. 2008; 15(3): 154-163, doi: 10.1002/cpp.574, indexed in Pubmed: 19115436.

3. Eckersley R. Troubled youth: an island of misery in an ocean of happiness, or the tip of an iceberg of suffering? Early Interv Psychiatry. 2011; 5 Suppl 1: 6-11, doi: 10.1111/j.1751-7893.2010.00233.x, indexed in Pubmed: 21208384.

4. Bor W, Dean AJ, Najman J, et al. Are child and adolescent mental health problems increasing in the 21st century? A systematic review. Aust N Z J Psychiatry. 2014; 48(7): 606-616, doi: 10.1177/0004867414533834, indexed in Pubmed: 24829198.

5. Collishaw S. Annual research review: Secular trends in child and adolescent mental health. J Child Psychol Psychiatry. 2015; 56(3): 370393, doi: 10.1111/jcpp.12372, indexed in Pubmed: 25496340.

6. Rozanov VA. Evolution of children and adolescents mental health for the last decades: an attempt of objective evaluation based on different approaches. Sotsialnaya I Klinichaskaya Psychiatriya [Social and Clinical Psychiatry], 2018; 28(1): 62-73. [Article in Russian].

7. Twenge JM, Gentile B, DeWall CN, et al. Birth cohort increases in psychopathology among young Americans, 1938-2007: A cross -temporal meta-analysis of the MMPI. Clin Psychol Rev. 2010; 30(2): 145-154, doi: 10.1016/j.cpr.2009.10.005, indexed in Pubmed: 19945203.

8. Apter A, Bursztein C, Bertolote J, et al. Suicide on all the continents in the young. Oxford Textbook of Suicidology and Suicide Prevention. 2009: 622-628, doi: 10.1093/med/9780198570059.003.0085.

9. Rozanov VA. [Suicides among children and adolescents - what is going on and what is the reason? ], Suitsidologia [Suicidology], 2014; 5(4): 16-31 [Article in Russian].

10. Rozanov VA. Stress and epigenetics in suicide. Academic Press, London 2017: 227.

11. Schoenbaum M, Kessler RC, Gilman SE, et al. Army STARRS Collaborators. Predictors of suicide and accident death in the Army Study to Assess Risk and Resilience in Servicemembers (Army STARRS): results from the Army Study to Assess Risk and Resilience in Servicemembers (Army STARRS). JAMA Psychiatry. 2014; 71(5): 493-503, doi: 10.1001/jamapsychiatry.2013.4417, indexed in Pubmed: 24590048.

12. Kessler RC, Heeringa SG, Stein MB, et al. Army STARRS Collaborators. Thirty-day prevalence of DSM-IV mental disorders among nondeployed soldiers in the US Army: results from the Army Study to Assess Risk and Resilience in Servicemembers (Army STARRS). JAMA Psychiatry. 2014; 71(5): 504-513, doi: 10.1001/jamapsychiatry.2014.28, indexed in Pubmed: 24590120.

13. Perry M. The recruitment problem the military doesn't want to talk about. The American Conservative. Available at. https://www. theamericanconservative.com/articles/the-recruitment-problem-the -military-doesnt-want-to-talk-about/. 
14. Shamrey VK. editor. Psychiatriya Voyn I Katastroph [Psychiatry of Wars and Katastrophs]. SpetsLit, Saint Petersburg 2015: 620.

15. Hyams KC. Mental health screening before troop deployment: is not supported by current evidence. BMJ. 2006; 333(7576): 979-980, doi: 10.1136/bmj.39023.648970.80, indexed in Pubmed: 17095759.

16. Rona RJ, Hooper R, Jones M, et al. Mental health screening in armed forces before the Iraq war and prevention of subsequent psychological morbidity: follow-up study. BMJ. 2006; 333(7576): 991, doi: 10.1136/bmj.38985.610949.55, indexed in Pubmed: 17023434.

17. Rozanov VA, Mehlum L, Stiliha R. Suicide in military settings. Combatants and veterans. In: Wasserman D, Wasserman C, editors. Oxford Textbook on Suicidology and Suicide Prevention, A global Perspective. Oxford University Press, Oxford 2009: 257-265.

18. Allen JP, Cross G, Swanner J. Suicide in the Army: a review of current information. Mil Med. 2005; 170(7): 580-584, doi: 10.7205/ milmed.170.7.580, indexed in Pubmed: 16130637.

19. Micklewright S. Deliberate self harm in the Royal Navy. An audit of cases presenting to the Department of Community Mental Health, RH Haslar between 1 March 1999 and 28 February 2002. J R Nav Med Serv. 2005; 91(1): 12-25, indexed in Pubmed: 15986836.

20. DASA. Suicide and open verdict death in the UK regular Armed Forces 1984-2006. 2007; Available at. http://www.DASA.mod.uk.

21. Sharaevskiy GY, Kolkutin VV, Fetisov VA. Medical-forensic evaluation of deaths from external causes in the Navy. Voenno-Meditsinskiy Zhurnal. Military-Medical J. 2002; 323: 7-10. [Aricle in Russian].

22. Alderton T, Bloor T, Kahveci M, et al. The Global Seafarer: Living and working conditions in a globalized industry. International Labour Office, Geneva. 2004: ILO.

23. Lefcowitz RY, Slade MD. Seafarers Mental Health Study. Final Report. ITF Seafarers' Trust \& Yale University. 2019.

24. Mellbye A, Carter T. Seafarers' depression and suicide. Int Marit Health. 2017; 68(2): 108-114, doi: 10.5603/IMH.2017.0020, indexed in Pubmed: 28660614.

25. Roberts SE. Surveillance of work related mortality among seafarers employed on board Isle of Man registered merchant ships from 1986 to 2005. Int Marit Health. 2006; 57(1-4): 9-23, indexed in Pubmed: 17312690.

26. Roberts SE, Carter T. Mortality from accidents, disease, suicide and homicide in the British fishing industry from 1900 to 2010. Int Marit Health. 2015; 66(4): 211-219, doi: 10.5603/IMH.2015.0042, indexed in Pubmed: 26726892.

27. Oldenburg M, Jensen $\mathrm{HJ}$, Wegner R. Burnout syndrome in seafarers in the merchant marine service. Int Arch Occup Environ Health. 2013; 86(4): 407-416, doi: 10.1007/s00420-012-0771-7, indexed in Pubmed: 22526089.

28. Szymańska K, Jaremin B, Rosik E. Suicides among Polish seamen and fishermen during work at sea. Int J Marit Med Health. 2006; 57(1-4): 36-45.
29. Rydstedt LW, Lundh M. Work demands are related to mental health problems for older engine room officers. Int Marit Health. 2012; 63(4): 176-180, indexed in Pubmed: 24595972.

30. Rydstedt LW, Lundh M. An ocean of stress? The relationship between psychosocial workload and mental strain among engine officers in the Swedish merchant fleet. Int Marit Health. 2010; 62(3): 168-175, indexed in Pubmed: 21154304.

31. Hjarnoe L, Leppin A. A risky occupation? (Un)healthy lifestyle behaviors among Danish seafarers. Health Promot Int. 2014; 29(4): 720-729, doi: 10.1093/heapro/dat024, indexed in Pubmed: 23630132.

32. Slišković A, Penezić Z. Lifestyle factors in Croatian seafarers as relating to health and stress on board. Work. 2017; 56(3): 371-380, doi: 10.3233/WOR-172501, indexed in Pubmed: 28339415.

33. Koester T. Human factors and everyday routine in the maritime work domain In: de Waard D, Brookhuis KA, Moraal J, Toffetti A. Human Factors in Transportation, Communication, Health, and the Workplace. Shaker, Maastricht 2002: 361.

34. Jones PB. Adult mental health disorders and their age at onset. Br J Psychiatry. 2018; 202(s54): s5-s10, doi: 10.1192/bjp. bp.112.119164.

35. Myers DG. The American Paradox: Spiritual Hunger in an Age of Plenty. Yale University Press, New Haven 2001: 431.

36. Putnam RD. Bowling Alone: The Collapse and Revival of American Community. Simon \& Schuster, New York 2000: 545.

37. Chaturvedi S, Prasad K, Angothu H, et al. How are social changes in the twenty first century relevant to mental health? Ind J Soc Psychiatry. 2016; 32(3): 227, doi: 10.4103/0971-9962.193195.

38. Friedli L. Mental health, resilience and inequalities. WHO, Geneva 2009: 55.

39. Trushchelev SA. [Improvement of defense of psychic health of military servicemen]. Voenno-Meditsinskiy Zhurnal [Military-Medical Journa]. 2010; 331(2): 39-41. [Article in Russian].

40. Nowosielski R, Mazurek T, Florkowski A. [Psychopathology service on ships]. (article in Polish]. Polski Merkuriusz Lekarski. 2010; 28(168): 513-515.

41. Charney DS. Psychobiological mechanisms of resilience and vulnerability: implications for successful adaptation to extreme stress. Am J Psychiatry. 2004; 161(2): 195-216, doi: 10.1176/appi. ajp.161.2.195, indexed in Pubmed: 14754765.

42. Gunnar MR, Frenn K, Wewerka SS, et al. Moderate versus severe early life stress: associations with stress reactivity and regulation in 10-12-year-old children. Psychoneuroendocrinology. 2009; 34(1): 62-75, doi: 10.1016/j.psyneuen.2008.08.013, indexed in Pubmed: 18835102.

43. McMenemy L, Bennett PM, Stapley SA. The Ship's Doctor in the Royal Navy-100 years of tradition and progress. J R Nav Med Serv. 2015; 101(1): 15-19, indexed in Pubmed: 26292387.

44. Morrow L, Abrams C, Shaw A. Creating a Healthy Ship. J R Nav Med Serv. 2017; 103(1): 5-9, indexed in Pubmed: 30088730. 